\title{
Modular corneal surgery
}

\section{Eye (2006) 20, 519-520. doi:10.1038/sj.eye.6701904}

In this issue Noble and colleagues report their early experience with modular corneal surgery. What is modular corneal surgery? Our understanding of corneal function has now led to an understanding that individual surgical sites can be addressed rather than involving unnecessary structures. Thus, corneal surgery is now even more NOT just penetrating keratoplasty (PK).

The article by Noble specifically addresses one of the most important indications for corneal surgery: keratoconus (KC). Traditionally managed by contact lenses with all their problems of tolerance and risks of complications, surgery was regarded as something to be avoided if possible. The main reasoning was that IF irreversible endothelial rejection occurred, the patient was substantially worse off. While such cases are mercifully rare, most corneal surgeons will have or have come across such patients. Why transplant the endothelium? Conventional lamellar keratoplasty has a reputation for equivocal visual results; 6/9 perhaps, $6 / 12$ probably: unlikely to satisfy young probably dynamic demanding individuals. Thus the desire to have something better: deep lamellar keratoplasty (DLK). Where did the impetus come from? I think the answer is obvious as I see many patients with $6 / 6$ vision or better who have had lamellar corneal surgery. The fact that it is not widely available within the NHS should not allow us to ignore the very real advances in the understanding and technology that have resulted from corneal refractive surgery. Although in training I was told by a Professor of Ophthalmology that it was only for 'cowboys', it has clearly contributed to our scientific understanding of the physiology of the cornea and physiological optics of the eye. The use of wave-front guided treatments can, in some cases, achieve visual acuities that exceed those found in normal eyes. While such visual achievements cannot yet be
C Claoué

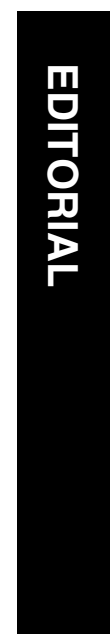

achieved either in all eyes or in pathological corneas the scope for such achievement is there.

DLK is a demanding technique and even in the best hands has a small incidence of conversion to PK. Nevertheless, the visual acuities approach those of PK and the advantage in the loss of risk of endothelial rejection is enormous. As dedicated corneal surgeons become more comfortable with the technique it seems likely that it will be offered to patients with $\mathrm{KC}$ at an earlier stage in the disease process, hopefully reducing the burden of CL-related corneal complications.

Other forms of modular corneal surgery are in development. Pseudophakic bullous keratopathy and decompensated Fuchs' endothelial dystrophy are currently treated by PK but solely to transplant endothelium. If it were possible to transplant endothelium and leave the stroma and surface untouched all the postkeratoplasty problems with astigmatism and suture-related complications should be substantially reduced. This would mean that the tedious delay in visual rehabilitation in patients who are frequently elderly should be much improved. The described procedure of deep lamellar endothelial keratoplasty (DLEK) is beginning to justify these aspirations although the procedure is not yet widely practised. Again, a very high level of surgical expertise is required.

What other forms of modular corneal surgery can be envisaged? Automated Homologous Lamellar Keratoplasty involves the use of a LASIK microkeratome to remove opacities in the anterior stroma and the replacement of stroma with a donor lenticule cut in likefashion. Since these eyes have structurally normal stroma (in contrast to $\mathrm{KC}$ ) the technical demands of DLK are not required. A further example of modular corneal surgery is Limbal Stem Cell Grafting. We now appreciate that the limbal stem cells are critical in maintaining the critical refractive (and therefore visual) surface
Whipps Cross University Hospital, London, UK

Correspondence: Charles Claoué, Ophthalmology, Harold Wood Hospital, Gubbinsn Lane, Romford, UK

Tel: + 441708708454 ;

E-mail: charles@

dbcg.co.uk
Fax: + 441708708457 
of the eye, and specific insults to this fragile cell population can now be addressed specifically.

I do not propose to try to foretell the future of corneal surgery, but clearly the future is likely to be more complex surgically than the past, and I think that more corneal surgery is likely to be performed and the increasing majority by dedicated corneal surgeons. Noble et al are to be commended for helping us along this path. 